\title{
Sobolev Spaces and Potential Spaces Associated to Hermite Polynomials Expansions
}

\author{
IRIS A. LÓPEz P. \\ Universidad Simón Bolivar, Departamento de Matemáticas Puras y Aplicadas, \\ Aptd 89000, Caracas 1080-A, Venezuela \\ iathamaica@usb.ve
}

Presented by Carmen Calvo

Received June 14, 2017

\begin{abstract}
The aim of this paper is to study the relation existing between potential spaces and Sobolev spaces, induced by the Ornstein-Uhlenbeck differential operator and associated to Hermite polynomials expansions, where we consider the multidimensional Gaussian measure. By means of analytical methods we prove that potential spaces and Sobolev spaces are spaces with equivalent norms.
\end{abstract}

Key words: Hermite polynomials, Gaussian measure, Sobolev spaces, potential spaces, Meyer's multiplier theorem.

AMS Subject Class. (2000): 42C10, 26A33.

\section{INTRODUCTION}

In the classical harmonic analysis theory the study of the differentiability and smoothness of functions, $f: \mathbb{R}^{d} \rightarrow \mathbb{R}$, can be described in the context of Banach spaces of functions. Thus, Sobolev spaces $W_{k}^{p}\left(\lambda_{d}\right)$ and potential spaces $L_{s}^{p}\left(\lambda_{d}\right)$ are taking into consideration, where we denote $\lambda_{d}$ as the Lebesgue measure, $s>0, k \in \mathbb{N}$ and $1<p<\infty$. On the one hand, Sobolev spaces $W_{k}^{p}\left(\lambda_{d}\right)$ allows us to consider functions, such that, $f \in L^{p}\left(\lambda_{d}\right)$ and its partial derivatives $\partial^{\alpha} f \in L^{p}\left(\lambda_{d}\right)$, with $|\alpha| \leq k$, where the derivatives are understood in a suitable weak sense to make the space complete. On the other hand, potential spaces $L_{s}^{p}\left(\lambda_{d}\right)$ are defined by using fractional powers of the Laplacean $(-\triangle)^{-s / 2}, 0<s<d$, and its variants $(I-\triangle)^{-s / 2}, s>0$. These fractional powers are known as Riesz potentials and Bessel potentials respectively and we have that the potential spaces $L_{s}^{p}\left(\lambda_{d}\right)$ are subspaces of $L^{p}\left(\lambda_{d}\right)$, such that, $f=(I-\triangle)^{-s / 2} g$, with $g \in L^{p}\left(\lambda_{d}\right)$. Riesz potentials, Bessel potentials and their properties have been deeply studied and by means of these operators it has been obtained that

$$
W_{k}^{p}\left(\lambda_{d}\right)=L_{k}^{p}\left(\lambda_{d}\right) \quad \text { with } \quad k \in \mathbb{N} \quad \text { and } \quad 1<p<\infty,
$$


(see $[15,6])$. This identity is very meaningful because the condition defining to $W_{k}^{p}\left(\lambda_{d}\right)$ spaces is not easy to check for any function given, nevertheless, the condition defining to $L_{k}^{p}\left(\lambda_{d}\right)$ can be described in terms of Bessel potentials which have integral representations.

Similar identity holds in the Hermite setting and a probabilistic proof of this fact has been given by H. Sugita in [16] and S. Watanabe in [17], where the notion of Sobolev spaces of Wiener functionals has been introduced to develop Malliavin's calculus. This question has been studied by B. Bongioanni and J. L Torrea in $[1,2]$, where they considered Sobolev spaces associated to Hermite functions expansions and Laguerre functions expansions. However, in [7] we introduced the fractional derivative for the gaussian measure $\gamma_{d}$ and by means of analytical methods we obtained that

$$
W_{k}^{p}\left(\gamma_{1}\right)=L_{k}^{p}\left(\gamma_{1}\right), \quad \text { with } \quad k \in \mathbb{N} \quad \text { and } \quad 1<p<\infty,
$$

but only in the unidimensional case.

Therefore, the purpose of this paper is to extend this identity to the multidimensional case. If $d \geq 1, k \in \mathbb{N}$ and $1<p<\infty$, we shall prove that

$$
W_{k}^{p}\left(\gamma_{d}\right)=L_{k}^{p}\left(\gamma_{d}\right)
$$

and once more, Bessel potentials and adjoint Gaussian-Riesz transforms are key tools in the proof of this fact. In the Laguerre polynomials and Jacobi polynomials setting, some similar results have been obtained in [5] and [8], respectively. Particularly, if $1<p<\infty, 0<s<1$ and $k \geq 1$ we obtain that $W_{k}^{p}\left(\gamma_{d}\right) \subset L_{s}^{p}\left(\gamma_{d}\right)$ and the inclusion is proper.

The paper is organized as follows. Section 2 contains some basic facts notation and we obtain the result of this paper in Section 3.

\section{Preliminary definitions.}

Let $\beta=\left(\beta_{1}, \ldots, \beta_{d}\right) \in \mathbb{N}^{d} \cup\{\overrightarrow{0}\}$ be a multi-index, so $\beta !=\prod_{i=1}^{d} \beta_{i}$ ! and $|\beta|=\sum_{i=1}^{d} \beta_{i}$. Let us denote by $\partial_{i}=\frac{\partial}{\partial x_{i}}$, for each $1 \leq i \leq d$, and $\partial^{\beta}=\partial_{1}^{\beta_{1}} \cdots \partial_{d}^{\beta_{d}}$. Let $\mathcal{P}$ be the subspace of polynomials functions on $\mathbb{R}^{d}$.

We denote the Gaussian measure $\gamma_{d}(d x)=\frac{e^{-|x|^{2}}}{\pi^{d / 2}} d x$, with $x \in \mathbb{R}^{d}$ and the Ornstein-Uhlenbeck differential operator is defined as

$$
L=\frac{1}{2} \triangle_{x}-\left\langle x, \nabla_{x}\right\rangle
$$


Let us consider the normalized Hermite polynomials of order $\beta$, in $d$ variables, defined as

$$
h_{\beta}(x)=\frac{1}{\left(2^{|\beta|} \beta !\right)^{1 / 2}} \prod_{i=1}^{d}(-1)^{\beta_{i}} e^{x_{i}^{2}} \frac{\partial^{\beta_{i}}}{\partial x_{i}^{\beta_{i}}}\left(e^{-x_{i}^{2}}\right),
$$

with $h_{0}(x)=1$, (see [18, pages 105-106]) and it is well known that the Hermite polynomials are eigenfunctions of $L$; this way,

$$
L h_{\beta}(x)=-|\beta| h_{\beta}(x) .
$$

Also,

$$
\partial_{j} h_{\beta}(x)=\sqrt{2 \beta_{j}} h_{\beta-e_{j}}, \quad \text { for each } \quad 1 \leq j \leq d
$$

and if $\alpha$ is a multi-index,

$$
\partial^{\alpha} h_{\beta}(x)=\left\{\begin{array}{lr}
2^{|\alpha| / 2}\left(\prod_{j=1}^{d} \beta_{j}\left(\beta_{j}-1\right) \cdots\left(\beta_{j}-\alpha_{j}+1\right)\right)^{1 / 2} h_{\beta-\alpha}(x), & \text { if } \alpha_{j} \leq \beta_{j} \\
0, & \forall j=1, \ldots, d \\
\text { otherwise }
\end{array}\right.
$$

Now, given a function $f \in L^{1}\left(\gamma_{d}\right)$ its $\beta$-Fourier-Hermite coefficient is defined by

$$
c_{\beta}^{f}=\int_{\mathbb{R}^{d}} f(x) h_{\beta}(x) \gamma_{d}(d x)
$$

and let $C_{n}$ be the closed subspace of $L^{2}\left(\gamma_{d}\right)$ generated by the linear combinations of $\left\{h_{\beta}:|\beta|=n\right\}$. The orthogonality of the Hermite polynomials with respect to $\gamma_{d}$, lets us see that $\left\{C_{n}\right\}$ is an orthogonal decomposition of $L^{2}\left(\gamma_{d}\right)$

$$
L^{2}\left(\gamma_{d}\right)=\bigoplus_{n=0}^{\infty} C_{n}
$$

which is called the Wiener chaos decomposition, see [17]. Now, we denote $J_{n}$ the orthogonal projection of $L^{2}\left(\gamma_{d}\right)$ onto $C_{n}$. If $f \in L^{2}\left(\gamma_{d}\right)$, we have that

$$
J_{n} f=\sum_{|\beta|=n} c_{\beta}^{f} h_{\beta}
$$

and its Hermite expansion is given by $f=\sum_{n \geq 0} J_{n} f$. Then, following [16] there exists a positive constant $C_{p, n}$, such that,

$$
\left\|J_{n} f\right\|_{p, \gamma_{d}} \leq C_{p, n}\|f\|_{p, \gamma_{d}} \quad \text { for } \quad 1<p<\infty
$$


Also, if $f \in L^{2}\left(\gamma_{d}\right)$ the operator

$$
L f=\sum_{n \geq 0}-n J_{n} f
$$

defined on the domain $D_{2}(L)=\left\{f \in L^{2}\left(\gamma_{d}\right): \sum_{n \geq 0} \sum_{|\beta|=n}\left|c_{\beta}^{f}\right|^{2}<\infty\right\}$ is a self-adjoint extension of $L$ considered on dense subspace of $L^{2}\left(\gamma_{d}\right)$. More precisely, $L$ has a clousure wich also will denote by $L$.

In this context, the Ornstein-Uhlenbeck semigroup, $\left\{T_{t}\right\}_{t \geq 0}$, is defined as

$$
T_{t} f(x)=\frac{1}{\left(1-e^{-2 t}\right)^{d / 2}} \int_{\mathbb{R}^{d}} e^{-\frac{e^{-2 t}\left(|x|^{2}+|y|^{2}-2 e^{-t}\langle x, y\rangle\right.}{1-e^{-2 t}}} f(y) \gamma_{d}(d y),
$$

where its kernel is given by Mehler formula

$$
\frac{1}{\left(1-e^{-2 t}\right)^{d / 2}} e^{-\frac{e^{-2 t}\left(|x|^{2}+|y|^{2}\right)-2 e^{-t}\langle x, y\rangle}{1-e^{-2 t}}}=\sum_{n \geq 0} \sum_{|\beta|=n} e^{-|\beta| t} h_{\beta}(x) h_{\beta}(y) .
$$

It is well known that $\left\{T_{t}\right\}_{t>0}$ is a symmetric diffusion semigroup, with infinitesimal generator $L$, see $[14,16]$ and moreover

$$
\left\|T_{t}\left(I-J_{0}-\cdots-J_{n-1}\right) f\right\|_{p, \gamma_{d}} \leq C_{p, n} e^{-n t}\|f\|_{p, \gamma_{d}}, \quad 1<p<\infty .
$$

By means of Bochner subordination formula the Poisson-Hermite semigroup $\left\{P_{t}\right\}_{t \geq 0}$ is defined as

$$
P_{t} f(x)=\frac{1}{\sqrt{\pi}} \int_{0}^{\infty} \frac{e^{-u}}{\sqrt{u}} T_{t^{2} / 4 u} f(x) d u
$$

and similarly, $\left\{P_{t}\right\}_{t \geq 0}$ is a strongly continuous semigroup on $L^{p}\left(\gamma_{d}\right)$ with infinitesimal generator $(-L)^{1 / 2}$, (see [14]). From (2.1) we obtain that

$$
T_{t} h_{\beta}(x)=e^{-t|\beta|} h_{\beta}(x) \quad \text { and } \quad P_{t} h_{\beta}(x)=e^{-t \sqrt{|\beta|}} h_{\beta}(x)
$$

Moreover,

$$
T_{t}\left(J_{n} f\right)=e^{-n t} J_{n} f \quad \text { and } \quad P_{t}\left(J_{n} f\right)=e^{-\sqrt{n} t} J_{n} f .
$$

Now, if $s>0$, similar to the classical case, the gaussian fractional integral of order $s, I_{s}^{\gamma}$, is defined by

$$
I_{s}^{\gamma}:=(-L)^{-s / 2} \Pi_{0}, \quad \text { where } \quad \Pi_{0}=I-J_{0} .
$$


This (formal) definition is correct for all Hermite polynomials $h_{\beta}$, since by using (2.1) we have that

$$
I_{s}^{\gamma} h_{\beta}(x)=\frac{1}{|\beta|^{s / 2}} h_{\beta}(x), \quad \forall|\beta|>0,
$$

and define $I_{s}^{\gamma} h_{0}(x)=0$, see $[3,13]$. In the case that $f \in L^{1}\left(\gamma_{d}\right)$, an integral representation of $I_{s}^{\gamma}$ is obtained in [13], which is given by

$$
I_{s}^{\gamma} f=\frac{1}{\Gamma(s)} \int_{0}^{\infty} t^{s-1} P_{t}\left(I-J_{0}\right) f d t
$$

By using (2.5), we can see that (2.8) coincides with (2.7), if $f=h_{\beta}, \forall|\beta|>0$ and consequently, (2.8) coincides with (2.7) if $f$ is a nonconstant polynomial or $f \in L^{2}\left(\gamma_{d}\right)$.

Again, from (2.3) and (2.4) we obtain that

$$
\left\|P_{t}\left(I-J_{0}\right) f\right\|_{p, \gamma_{d}} \leq C_{p} e^{-t}\|f\|_{p, \gamma_{d}} \quad 1<p<\infty,
$$

since

$$
e^{-t}=\frac{1}{\sqrt{\pi}} \int_{0}^{\infty} \frac{e^{-u}}{\sqrt{u}} e^{-t^{2} / 4 u} d u .
$$

Therefore, from (2.8) and (2.9) we can conclude

$$
\left\|I_{s}^{\gamma} f\right\|_{p, \gamma_{d}} \leq C_{p}\|f\|_{p, \gamma_{d}} \quad 1<p<\infty .
$$

Now, for $\alpha \in \mathbb{N}^{d}$, we consider the Riesz transform of order $|\alpha|$, associated to $L$, defined as, (see [10])

$$
R_{|\alpha|}^{\alpha}:=\partial^{\alpha} I_{|\alpha|}^{\gamma}
$$

and if $f \in L^{1}\left(\gamma_{d}\right)$ with $c_{0}^{f}=0$, then

$$
\begin{aligned}
& R_{|\alpha|}^{\alpha} f(x) \\
= & C_{d, \alpha} \int_{\mathbb{R}^{d}} \int_{0}^{1} r^{|\alpha|-1}\left(\frac{-\log r}{1-r^{2}}\right)^{\frac{|\alpha|-2}{2}} h_{\alpha}\left(\frac{y-r x}{\sqrt{1-r^{2}}}\right) \frac{e^{-\frac{|y-r x|^{2}}{1-r^{2}}}}{\left(1-r^{2}\right)^{d / 2+1}} d r f(y) d y .
\end{aligned}
$$

$L^{p}\left(\gamma_{d}\right)$ estimates for $1<p<\infty$ of the Gaussian-Riesz transform, have been showed by several authors using probabilistic and analytic methods (see for 
example $[4,10,11]$ among other authors). Particularly, if $f=h_{\beta}$, for each $i=1, \ldots, d$ and $\beta_{i} \geq \alpha_{i}$, we have

$$
R_{|\alpha|}^{\alpha} h_{\beta}(x)=\left(\frac{2^{|\alpha|}}{|\beta|^{|\alpha|}}\right)^{1 / 2}\left[\prod_{i=1}^{d} \beta_{i}\left(\beta_{i}-1\right) \cdots\left(\beta_{i}-\alpha_{i}+1\right)\right]^{1 / 2} h_{\beta-\alpha}(x)
$$

and the j-th Gaussian-Riesz transform of first order, $R_{1}^{j}=\partial_{j} I_{1}^{\gamma}, j=1, \ldots, d$, with respect to Hermite polynomials can be expressed as

$$
R_{1}^{j} h_{\beta}=\sqrt{\frac{2 \beta_{j}}{|\beta|}} h_{\beta-e_{j}}
$$

In [7], the j-th adjoint operator, $\left(R_{1}^{j}\right)^{*}$ of the Gaussian-Riesz transform $R_{1}^{j}$, has been defined as

$$
\left\langle\left(R_{1}^{j}\right)^{*} f, g\right\rangle_{\gamma_{d}}=\left\langle f, R_{1}^{j} g\right\rangle_{\gamma_{d}}
$$

and we can observe that $I_{1}^{\gamma}=(-L)^{-1 / 2}$ is a self-adjoint operator, then integrating by parts with respect $x_{j}$, we obtain

$$
\left\langle f, R_{1}^{j} g\right\rangle_{\gamma_{d}}=\left\langle f, \partial_{j} I_{1}^{\gamma} g\right\rangle_{\gamma_{d}}=\left\langle\delta_{j} f, I_{1}^{\gamma} g\right\rangle_{\gamma_{d}}=\left\langle I_{1}^{\gamma} \delta_{j} f, g\right\rangle_{\gamma_{d}},
$$

where $\delta_{j}(\cdot)=-\partial_{j}(\cdot)+2 x_{j}(\cdot)$. This way, we can express, for each $j=1, \ldots, d$,

$$
\left(R_{1}^{j}\right)^{*}:=I_{1}^{\gamma} \delta_{j}=(-L)^{-1 / 2} \delta_{j} .
$$

By means of the identity

$$
\sqrt{2\left(\beta_{j}+1\right)} h_{\beta+e_{j}}-2 x_{j} h_{\beta}+\sqrt{2 \beta_{j}} h_{\beta-e_{j}}=0,
$$

where $h_{-e_{j}}=0, \forall j=1, \ldots, d$, (see [18, pages 105-106]), we have that

$$
\delta_{j} h_{\beta}=\sqrt{2\left(\beta_{j}+1\right)} h_{\beta+e_{j}}
$$

and therefore,

$$
\left(R_{1}^{j}\right)^{*} h_{\beta}=\sqrt{\frac{2\left(\beta_{j}+1\right)}{|\beta|+1}} h_{\beta+e_{j}} .
$$

Also, in [7] we obtain the boundedness of $\mathrm{j}$-th adjoint operator of the Gaussian-Riesz transform $\left(R_{1}^{j}\right)^{*}$ for $1<p<\infty$. This follows easily from Hölder's inequality and the $L^{p}\left(\gamma_{d}\right)$ continuity of the Riesz transform. So,

$$
\left\|\left(R_{1}^{j}\right)^{*} f\right\|_{p, \gamma_{d}}=\operatorname{Sup}_{\|g\|_{q} \leq 1}\left|\left\langle f, R_{1}^{j} g\right\rangle_{\gamma_{d}}\right| \leq C_{q}\|f\|_{p, \gamma_{d}} .
$$


Similarly, the j-th adjoint Gaussian-Riesz operator $\left(R_{k}^{j}\right)^{*}$ of higher order $k$, with $k \geq 1$, is defined by

$$
\left(R_{k}^{j}\right)^{*}:=I_{k}^{\gamma} \delta_{j}^{k}=(-L)^{-k / 2} \delta_{j}^{k}, \quad \text { for each } j=1, \ldots, d,
$$

If $\alpha \in \mathbb{N}^{d}$, we can define the higher order adjoint operator of the Riesz transform by

$$
\left(R_{|\alpha|}^{\alpha}\right)^{*}:=I_{|\alpha|}^{\gamma} \delta_{d}^{\alpha_{d}} \circ \cdots \circ \delta_{1}^{\alpha_{1}}
$$

thus, we get

$$
\left(R_{|\alpha|}^{\alpha}\right)^{*} h_{\beta}(x)=\left(\frac{2^{|\alpha|}}{|\beta+\alpha|^{|\alpha|}}\right)^{1 / 2}\left[\prod_{i=1}^{d}\left(\beta_{i}+1\right) \cdots\left(\beta_{i}+\alpha_{i}\right)\right]^{1 / 2} h_{\beta+\alpha}(x)
$$

and if $1<p<\infty$ with $f \in L^{p}\left(\gamma_{d}\right)$, we have that

$$
\left\|\left(R_{|\alpha|}^{\alpha}\right)^{*} f\right\|_{p, \gamma_{d}} \leq C_{p}\|f\|_{p, \gamma_{d}} .
$$

Now, in [7] the Gaussian fractional derivative of order $s>0, D_{s}^{\gamma}$, is defined formally as

$$
D_{s}^{\gamma}:=(-L)^{s / 2}
$$

and for Hermite polynomials, from (2.1), we have that

$$
D_{s}^{\gamma} h_{\beta}(x)=|\beta|^{s / 2} h_{\beta}(x) .
$$

Particularly, from (2.14), we have that $D_{s}^{\gamma} h_{0}(x)=0$ and, similar to the classical case, the Gaussian fractional derivative of a constant function is equal to zero, see $[14,12]$. In the case of $0<s<1$, we can write

$$
D_{s}^{\gamma} f=\frac{1}{c_{s}} \int_{0}^{\infty} t^{-s-1}\left(P_{t} f-f\right) d t
$$

where

$$
c_{s}=\int_{0}^{\infty} u^{-s-1}\left(e^{-u}-1\right) d u,
$$

for $f \in \mathcal{P}$ and $f \in L^{2}\left(\gamma_{d}\right)$ (see [7]). This way, (2.15) is an integral representation of $D_{s}^{\gamma} f$. By using (2.5) we get that (2.15) coincides with (2.14), if $f=h_{\beta}, \forall|\beta|>0$ and $0<s<1$. Similarly, by means of the property $P_{t} 1=1$ we conclude that (2.15) coincides with (2.14), if $f=h_{0}$. Moreover, if $f \in \mathcal{P}$, by $(2.7)$ and (2.14) we obtain that

$$
I_{s}^{\gamma}\left(D_{s}^{\gamma} f\right)=D_{s}^{\gamma}\left(I_{s}^{\gamma} f\right)=\Pi_{0} f .
$$


Following S. Watanabe [17] and H. Sugita [16], we consider the GaussianBessel potentials defined by

$$
(I-L)^{-s / 2} f=\sum_{n=0}^{\infty}(1+n)^{-s / 2} J_{n} f, \quad \text { for } f \in \mathcal{P} .
$$

By means of the Gamma function we obtain that

$$
\frac{1}{\Gamma(s / 2)} \int_{0}^{\infty} t^{\frac{s}{2}-1} e^{-(1+n) t} d t=(1+n)^{-s / 2},
$$

thus, (2.6) lets us write

$$
(I-L)^{-s / 2} f=\frac{1}{\Gamma(s / 2)} \int_{0}^{\infty} t^{\frac{s}{2}-1} e^{-t} T_{t} f d t, \quad \text { for } f \in \mathcal{P}
$$

and by use of the contraction property of the semigroup $\left\{T_{t}\right\}_{t \geq 0}$, we obtain that

$$
\left\|(I-L)^{-s / 2} f\right\|_{p, \gamma_{d}} \leq\|f\|_{p, \gamma_{d}} .
$$

Then, the Gaussian-Bessel potential spaces of order $s \geq 0, L_{s}^{p}\left(\gamma_{d}\right)$, with $1<$ $p<\infty$, can be defined as the completion of the polynomials with respect to the norm

$$
\|f\|_{p, s}:=\left\|(I-L)^{s / 2} f\right\|_{p, \gamma_{d}} ;
$$

in other words, $L_{s}^{p}\left(\gamma_{d}\right)$ is a subspace of $L^{p}\left(\gamma_{d}\right)$ consisting of all $f$ which can be written in the form

$$
f=(I-L)^{-s / 2} \psi, \quad \text { with } \quad \psi \in L^{p}\left(\gamma_{d}\right),
$$

where

$$
\|f\|_{p, s}=\|\psi\|_{p, \gamma_{d}} .
$$

These potential spaces present the following inclusion properties (see [7, 17] for more details).

i) If $p \leq q$ then $L_{s}^{q}\left(\gamma_{d}\right) \subseteq L_{s}^{p}\left(\gamma_{d}\right)$, for each $s>0$.

ii) If $0<s \leq r$ then $L_{r}^{p}\left(\gamma_{d}\right) \subseteq L_{s}^{p}\left(\gamma_{d}\right)$, for each $1<p<\infty$.

Then, in [7] the following Theorem has been obtained

Theorem 2.1. Let $s \geq 0$ and $1<p<\infty$. Then $f \in L_{s}^{p}\left(\gamma_{d}\right)$ if and only if $D_{s}^{\gamma} f \in L^{p}\left(\gamma_{d}\right)$. Moreover,

$$
B_{p, s}\|f\|_{p, s} \leq\left\|D_{s}^{\gamma} f\right\|_{p, \gamma_{d}} \leq A_{p, s}\|f\|_{p, s} .
$$


Particularly, we can observe that if $\left\{P_{n}\right\} \in \mathcal{P}$, such that, $\lim _{n \rightarrow \infty} P_{n}=f$ in $L_{s}^{p}\left(\gamma_{d}\right)$, then $\lim _{n} D_{s}^{\gamma} P_{n}$ exists in $L_{s}^{p}\left(\gamma_{d}\right)$ and does not depend on the choice of a sequence $\left\{P_{n}\right\}_{n}$.

Theorem 2.1 is a corollary of the following theorem, obtained by P.A. Meyer, see $[9,16]$. This theorem shall be an important tool to develop our result in Section 3.

Theorem 2.2. (Meyer's multiplier theorem) Let $T_{\phi}$ be given by

$$
T_{\phi}=\sum_{n \geq 0} \phi(n) J_{n}
$$

where $\{\phi(n)\}_{n>0}$ is a real sequence. Assume that $h(z)$ is a function, which is analytic on some neighborhood of the origin. If there are $n_{0} \in \mathbb{N}$, and a positive constant $s$, such that $h\left(n^{-s}\right)=\phi(n) \forall n \geq n_{0}$, then $T_{\phi}$ can uniquely extend to a bounded linear operator on $L^{p}\left(\gamma_{d}\right)$, for each $1<p<\infty$.

As an application of Meyer's multiplier theorem, in [7], the following result has been obtained.

Proposition 2.1. Given $1<p<\infty$ and $s \geq 1$. If $f \in L_{s}^{p}\left(\gamma_{d}\right)$, then $f \in L_{s-1}^{p}\left(\gamma_{d}\right)$ and for each $j=1, \ldots, d, \partial_{j} f \in L_{s-1}^{p}\left(\gamma_{d}\right)$. Moreover,

$$
\|f\|_{p, s-1}+\sum_{j=1}^{d}\left\|\partial_{j} f\right\|_{p, s-1} \leq A_{p, s, d}\|f\|_{p, s} .
$$

Finally, let us consider the Gaussian Sobolev space defined as

$$
W_{k}^{p}\left(\gamma_{d}\right):=\left\{f: \partial^{\alpha} f \in L^{p}\left(\gamma_{d}\right), \alpha \in \mathbb{N}^{d},|\alpha| \leq k\right\},
$$

where $\partial^{0} f=f$, equipped with the norm

$$
\|f\|_{W_{k}^{p}}:=\sum_{|\alpha| \leq k}\left\|\partial^{\alpha} f\right\|_{p, \gamma_{d}}
$$

(see [15, pages 121-122]; where $W_{k}^{p}\left(\lambda_{d}\right)$ spaces are considered).

Then for each $1<p<\infty$ and $k \geq m$, we can see that $\|f\|_{W_{m}^{p}} \leq\|f\|_{W_{k}^{p}}$ and therefore, $W_{k}^{p}\left(\gamma_{d}\right) \subseteq W_{m}^{p}\left(\gamma_{d}\right)$.

Also, from the definition of $W_{k}^{p}\left(\gamma_{d}\right)$ spaces we can see that $f \in W_{k}^{p}\left(\gamma_{d}\right)$, if and only if, $f$ and $\partial_{j} f \in W_{k-1}^{p}\left(\gamma_{d}\right)$ for each $j=1, \ldots, d$. Moreover, the two norms

$$
\|f\|_{W_{k}^{p}} \quad \text { and } \quad\|f\|_{W_{k-1}^{p}}+\sum_{j=1}^{d}\left\|\partial_{j} f\right\|_{W_{k-1}^{p}}
$$


are equivalent.

Remark. In [7], as has been mentioned previously, we proved that if $k \geq 1$, $1<p<\infty$ and $f \in L_{k}^{p}\left(\gamma_{1}\right)$, then there exist a positive constant, $B_{p, k}$, such that,

$$
\|f\|_{p, k} \leq B_{p, k}\left\|\frac{d^{k}}{d x^{k}} f\right\|_{p, \gamma_{1}}
$$

for the unidimensional case. Nevertheless, in order to motivate the results to be developed in Section 3, we recall how this inequality was proved.

In fact, if $f \in \mathcal{P}$ we define the operator $T_{k}$ as

$$
T_{k} f=\sum_{n \geq 0} \frac{(n+k)^{k}}{2^{k}(n+k) \cdots(n+1)} c_{n}^{f} h_{n} .
$$

Then using Meyer's Multipliers Theorem with the function

$$
h(z)=\frac{(1+k z)^{k}}{2^{k}(1+k z) \cdots(1+z)},
$$

we obtain that

$$
\left\|T_{k} f\right\|_{p, \gamma_{d}} \leq C_{p}\|f\|_{p, \gamma_{d}}
$$

Now, we introduce the operator $U_{k}$ as

$$
U_{k} h_{n}=\left(\frac{n+k}{2}\right)^{k / 2}((n+k) \cdots(n+1))^{-1 / 2} h_{n+k}
$$

and if $f \in \mathcal{P}$ we get that $U_{k} f=\sum_{n \geq 0} c_{n}^{f} U_{k}\left(h_{n}\right)$.

Denoting $\left(R_{k}\right)^{*}=(-L)^{-k / 2} \delta^{k}$ as the unidimensional adjoint Gaussian Riesz transform of order $k$, with $k \geq 1$, we can see that

$$
\left(R_{k}\right)^{*} h_{n}(x)=2^{k / 2} \frac{[(n+1) \cdots(n+k)]^{1 / 2}}{(n+k)^{k / 2}} h_{n+k} .
$$

Therefore, for each $k \geq 1$ and $n \geq 1$, we have

$$
U_{k}\left(h_{n}\right)=\left[\left(R_{k}\right)^{*} \circ T_{k}\right]\left(h_{n}\right) .
$$

For this reason, if $f \in \mathcal{P}$, by means of the $L^{p}\left(\gamma_{d}\right)$-continuity of the operators $\left(R^{k}\right)^{*}$ and $T_{k}$ we obtain that

$$
\left\|U_{k} f\right\|_{p, \gamma_{d}} \leq C_{p, k}\|f\|_{p, \gamma_{d}} .
$$


But by definition $\left(U_{k} \circ R^{k}\right)\left(h_{n}\right)=h_{n}$ and if $f \in \mathcal{P}$ with $c_{0}^{f}=0$, we get

$$
D_{k}^{\gamma} f=\left(U_{k} \circ R_{k} \circ D_{k}^{\gamma}\right) f=\left(U_{k} \circ \frac{d^{k}}{d x^{k}} \circ I_{k}^{\gamma} \circ D_{k}^{\gamma}\right) f=U_{k}\left(\frac{d^{k}}{d x^{k}} f\right) .
$$

Therefore, by using the Theorem 2.1 and (2.18) we have that

$$
\|f\|_{p, k} \leq B_{p, k}\left\|\frac{d^{k}}{d x^{k}} f\right\|_{p, \gamma_{1}}
$$

and we can use the density of the polynomials in $L^{p}\left(\gamma_{1}\right)$ in the general case.

Now, let us consider the multidimensional case with $d>1$ and we would like to repeat a similar argument. In this case, we could define the operators

$$
T_{\alpha}\left(h_{\beta}\right)=\frac{|\beta+\alpha|^{|\alpha|}}{2^{|\alpha|} \prod_{i=1}^{d}(|\beta|+1) \cdots\left(|\beta|+\alpha_{i}\right)} h_{\beta}
$$

and

$$
U_{\alpha}\left(h_{\beta}\right)=\left(\frac{|\beta+\alpha|}{2}\right)^{|\alpha| / 2}\left[\prod_{i=1}^{d}\left(\beta_{i}+1\right) \cdots\left(\beta_{i}+\alpha_{i}\right)\right]^{-1 / 2} h_{\beta+\alpha}
$$

for $\alpha \in \mathbb{N}^{d}$. Thus, if $f \in \mathcal{P}$ we introduce

$$
T_{\alpha} f=\sum_{n \geq 0} T_{\alpha}\left(J_{n} f\right), \quad \text { and } \quad U_{\alpha} f=\sum_{n \geq 0} U_{\alpha}\left(J_{n} f\right)
$$

and by using Meyer's Multipliers Theorem with

$$
h(z)=\frac{2^{-|\alpha|}(1+|\alpha| z)}{\prod_{i=1}^{d}\left(1+\alpha_{i} z\right) \cdots(1+z)} \quad \text { and } \quad \phi(|\beta|)=\frac{2^{-|\alpha|}\left(1+\frac{|\alpha|}{|\beta|}\right)}{\prod_{i=1}^{d}\left(1+\frac{1}{|\beta|}\right) \cdots\left(1+\frac{\alpha_{i}}{|\beta|}\right)},
$$

we obtain that $T_{\alpha}$ has a $L^{p}\left(\gamma_{d}\right)$-continuous extension. However, we can see that

$$
\left[\left(R_{|\alpha|}^{\alpha}\right)^{*} \circ T_{\alpha}\right]\left(h_{\beta}\right)=\left(\frac{|\beta+\alpha|^{|\alpha|}}{2^{|\alpha|}}\right)^{1 / 2}\left[\frac{\prod_{i=1}^{d}\left(\beta_{i}+1\right) \cdots\left(\beta_{i}+\alpha_{i}\right)}{\prod_{i=1}^{d}(|\beta|+1) \cdots\left(|\beta|+\alpha_{i}\right)}\right]^{1 / 2} h_{\beta+\alpha}
$$


and therefore

$$
U_{\alpha} f \neq\left[\left(R_{|\alpha|}^{\alpha}\right)^{*} \circ T_{\alpha}\right] f,
$$

if $f \in \mathcal{P}$. In consequence, this reasoning fails in the multidimensional case. This way, to prove that

$$
\|f\|_{p, k} \simeq\|f\|_{W_{k}^{p}}
$$

we need to developed a different argument.

Consequently, we are able to present the results of this paper in the following section.

\section{The RESUlts}

By using (2.11) and (2.16) we can write

$$
\partial^{\alpha} f=R_{|\alpha|}^{\alpha} D_{|\alpha|}^{\gamma} f
$$

if $f$ is a nonconstant polynomial. Then, by means of the $L^{p}\left(\gamma_{d}\right)$-continuity of the Gaussian-Riesz transform and Theorem 2.1, we get

$$
\left\|\partial^{\alpha} f\right\|_{p, \gamma_{d}} \leq C_{p,|\alpha|}\|f\|_{p,|\alpha|}
$$

and therefore, the density of the polynomials in $L_{|\alpha|}^{p}\left(\gamma_{d}\right)$, with $|\alpha| \leq k$, the fact that $\|f\|_{p,|\alpha|} \leq\|f\|_{p, k}$ and (3.1), allows us to conclude that

$$
\|f\|_{W_{k}^{p}} \leq C_{p . k}\|f\|_{p, k}
$$

Consequently,

$$
L_{k}^{p}\left(\gamma_{d}\right) \subseteq W_{k}^{p}\left(\gamma_{d}\right) \quad \text { for each } \quad k \in \mathbb{N} .
$$

Now, we shall prove the converse inequality in (3.2). First, we establish the following Lemma, where the relation between Riesz potentials and Bessel potentials are obtained in the gaussian context; see [3] for similar results and compare with [15, pages 133-134].

Lemma 3.1. Let $s \geq 0$ and $1<p<\infty$.

i) Suppose $f \in \mathcal{P}$, then there exists a constant $C_{p}>0$, such that,

$$
\left\|\left(D_{s}^{\gamma} \circ(I-L)^{-s / 2}\right) f\right\|_{p, \gamma_{d}} \leq C_{p}\|f\|_{p, \gamma_{d}} .
$$

ii) Suppose $f \in \mathcal{P}$, then we have

$$
\left\|\left((I-L)^{s / 2} \circ I_{s}^{\gamma}\right) f\right\|_{p, \gamma_{d}} \leq A_{p}\|f\|_{p, \gamma_{d}} .
$$


iii) There exists a pair of operators $T_{1}$ and $T_{2}$, which are bounded operators on $L^{p}\left(\gamma_{d}\right)$, so that

$$
(I-L)^{s / 2}=T_{1}+D_{s}^{\gamma} \circ T_{2} .
$$

Proof. Items i) and ii) have been obtained in [3]. However, we present it with details for the sake of completeness.

i) If $f \in \mathcal{P}$ then $f=\sum_{n \geq 0} J_{n} f$. Particularly, we observe that

$$
\left(D_{s}^{\gamma} \circ(I-L)^{-s / 2}\right) J_{0} f=c_{0}^{f}\left(D_{s}^{\gamma} \circ(I-L)^{-s / 2}\right) h_{0}=0
$$

and we can express

$$
\left(D_{s}^{\gamma} \circ(I-L)^{-s / 2}\right) f=\sum_{n \geq 0} \frac{n^{s / 2}}{(1+n)^{s / 2}} J_{n} f .
$$

Therefore, the result follows from Theorem 2.2 considering the function

$$
h(z)=(z+1)^{-s / 2} .
$$

ii) Again, we consider $f \in \mathcal{P}$, such that $f \in\left(C_{0}\right)^{\perp}$, then

$$
\left((I-L)^{s / 2} \circ I_{s}^{\gamma}\right) f=\sum_{n>0} \frac{(1+n)^{s / 2}}{n^{s / 2}} J_{n} f
$$

and similarly, by means of Meyer's multiplier theorem with the function

$$
h(z)=(z+1)^{s / 2},
$$

we get that

$$
\left\|\left((I-L)^{s / 2} \circ I_{s}^{\gamma}\right) f\right\|_{p, \gamma_{d}} \leq A_{p}\|f\|_{p, \gamma_{d}} .
$$

Particularly, if $f$ is a constant function, then $f=c_{0}^{f} h_{0}$ and since by definition $I_{s}^{\gamma} h_{0}=0$, we have that

$$
\left((I-L)^{s / 2} \circ I_{s}^{\gamma}\right) f=c_{0}^{f}\left((I-L)^{s / 2} \circ I_{s}^{\gamma}\right) h_{0}=0 .
$$

iii) We choose $T_{1}=I$ and

$$
T_{2}=\left((I-L)^{s / 2} \circ I_{s}^{\gamma}\right)-I_{s}^{\gamma} .
$$

Then if $f \in \mathcal{P}$, such that, $f \in\left(C_{0}\right)^{\perp}$, we obtain

$$
\left\|T_{2} f\right\|_{p, \gamma_{d}} \leq\left\|\left((I-L)^{s / 2} \circ I_{s}^{\gamma}\right) f\right\|_{p, \gamma_{d}}+\left\|I_{s}^{\gamma} f\right\|_{p, \gamma_{d}}
$$

and iii) follows from ii) and (2.10).

Particularly, if $f$ is a constant function, we have that $I_{s}^{\gamma} f=0$ and therefore, $T_{2} f=0$. 
Remark. It should be emphasized, that polynomials are dense in $L^{p}\left(\gamma_{d}\right)$, for $1<p<\infty$. This way, the Lemma 3.1 states that the operators defined by

$$
D_{s}^{\gamma} \circ(I-L)^{-s / 2} \text { and }(I-L)^{s / 2} \circ I_{s}^{\gamma},
$$

are bounded operators on $L^{p}\left(\gamma_{d}\right)$, on every $1<p<\infty$.

Now, on the one hand, by using (2.12) and (2.13) we observe that

$$
\left(R_{1}^{j}\right)^{*}\left(\partial_{j} h_{\beta}\right)=\left(R_{1}^{j}\right)^{*}\left(\sqrt{2 \beta_{j}} h_{\beta-e_{j}}\right)=\frac{2 \beta_{j}}{|\beta|^{1 / 2}} h_{\beta},
$$

for each $j=1, \ldots, d$ and $|\beta| \neq 0$. Thus,

$$
\sum_{j=1}^{d}\left(R_{1}^{j}\right)^{*}\left(\partial_{j} h_{\beta}\right)=2|\beta|^{1 / 2} h_{\beta}
$$

and if $g \in \mathcal{P}$, we obtain that

$$
\sum_{j=1}^{d}\left(R_{1}^{j}\right)^{*}\left(\partial_{j} J_{n} g\right)=2 n^{1 / 2} J_{n} g
$$

In consequence,

$$
D_{1}^{\gamma} g=\frac{1}{2} \sum_{j=1}^{d}\left(R_{1}^{j}\right)^{*}\left(\partial_{j} g\right) .
$$

Particularly, if $g$ is a constant function we can see that $D_{1}^{\gamma} g=0$ and since $\partial_{j} g=0$, then (3.4) is also true.

On other hand, if $s \geq 1$, we claim that

$$
(I-L)^{-(s-1) / 2}\left(\partial_{j} g\right)=\left(T_{0} \circ \partial_{j}(I-L)^{-(s-1) / 2}\right) g,
$$

where we set $T_{0}:=(I-L)^{(s-1) / 2} \circ I_{s-1}^{\gamma}$.

In fact, suppose $g \in \mathcal{P}$, such that, $g \in\left(C_{0}\right)^{\perp}$. Then $g=\sum_{n>0} J_{n} g$ and

$$
\partial_{j}(I-L)^{-(s-1) / 2} g=\sum_{n>0}\left(\frac{1}{n+1}\right)^{(s-1) / 2} \sum_{|\beta|=n} \sqrt{2 \beta_{j}} c_{\beta}^{g} h_{\beta-e_{j}} .
$$


Also,

$$
\begin{aligned}
(I-L)^{-(s-1) / 2} \partial_{j} g & =\sum_{n>0} \sum_{|\beta|=n} \frac{\sqrt{2 \beta_{j}} c_{\beta}^{g}}{|\beta|^{(s-1) / 2}} h_{\beta-e_{j}} \\
& =\sum_{n>0}\left(\frac{1+n}{n}\right)^{(s-1) / 2}\left(\frac{1}{1+n}\right)^{(s-1) / 2} \sum_{|\beta|=n} \sqrt{2 \beta_{j}} c_{\beta}^{g} h_{\beta-e_{j}} \\
& =\left((I-L)^{(s-1) / 2} \circ I_{s-1}^{\gamma} \circ \partial_{j}(I-L)^{-(s-1) / 2}\right) g
\end{aligned}
$$

which proves (3.5). Particularly, if $g$ is a constant function we have that

$$
(I-L)^{-(s-1) / 2}\left(\partial_{j} g\right)=0 \quad \text { and } \quad \partial_{j}(I-L)^{-(s-1) / 2} g=c_{0}^{g} \partial_{j} h_{0}=0
$$

and therefore, (3.5) is also true.

Then, we are able to prove the following proposition (see [15, pages $136-$ $138])$.

Proposition 3.1. Given $1<p<\infty$ and $s \geq 1$. Suppose that $f \in$ $L_{s-1}^{p}\left(\gamma_{d}\right)$ and $\partial_{j} f \in L_{s-1}^{p}\left(\gamma_{d}\right)$ for each $j=1, \ldots, d$. Then, $f \in L_{s}^{p}\left(\gamma_{d}\right)$ and also,

$$
\|f\|_{p, s} \leq B_{p, s}\left(\|f\|_{p, s-1}+\sum_{j=1}^{d}\left\|\partial_{j} f\right\|_{p, s-1}\right) .
$$

Proof. Let $f \in L_{s-1}^{p}\left(\gamma_{d}\right) \cap \mathcal{P}$. Then, there exists $\psi \in \mathcal{P}$, such that $f=$ $(I-L)^{-(s-1) / 2} \psi$ and therefore,

$$
f=\sum_{n \geq 0}\left(\frac{1}{n+1}\right)^{(s-1) / 2} J_{n} \psi
$$

and

$$
\partial_{j} f=\sum_{n \geq 0}\left(\frac{1}{n+1}\right)^{(s-1) / 2} \sum_{|\beta|=n} \sqrt{2 \beta_{j}} c_{\beta}^{\psi} h_{\beta-e_{j}} .
$$

Since $\psi \in L_{1}^{p}\left(\gamma_{d}\right) \cap \mathcal{P}$, we can write $\psi=(I-L)^{-1 / 2} h$ and according to the Lemma 3.1, part iii), where we consider $s=1, T_{1}=I$ and

$$
T_{2}=\left((I-L)^{1 / 2} \circ I_{1}^{\gamma}\right)-I_{1}^{\gamma},
$$


we obtain that

$$
h=(I-L)^{1 / 2} \psi=T_{1} \psi+\left(D_{1}^{\gamma} \circ T_{2}\right) \psi
$$

Therefore,

$$
\|h\|_{p, \gamma_{d}} \leq\left\|T_{1} \psi\right\|_{p, \gamma_{d}}+\left\|\left(D_{1}^{\gamma} \circ T_{2}\right) \psi\right\|_{p, \gamma_{d}} .
$$

Now, we can see that

$$
\|h\|_{p, \gamma_{d}}=\left\|(I-L)^{1 / 2} \psi\right\|_{p, \gamma_{d}}=\left\|(I-L)^{1 / 2}(I-L)^{(s-1) / 2} f\right\|_{p, \gamma_{d}}=\|f\|_{p, s}
$$

and

$$
\left\|T_{1} \psi\right\|_{p, \gamma_{d}}=\|\psi\|_{p, \gamma_{d}}=\left\|(I-L)^{(s-1) / 2} f\right\|_{p, \gamma_{d}}=\|f\|_{p, s-1} .
$$

On the other hand, by means of (3.4) we have that

$$
\left(D_{1}^{\gamma} \circ T_{2}\right) \psi=\left(T_{2} \circ D_{1}^{\gamma}\right) \psi=\frac{1}{2} T_{2}\left[\sum_{j=1}^{d}\left(R_{1}^{j}\right)^{*}\left(\partial_{j} \psi\right)\right]
$$

and therefore,

$$
\begin{aligned}
\left\|\left(D_{1}^{\gamma} \circ T_{2}\right) \psi\right\|_{p, \gamma_{d}} & =\frac{1}{2}\left\|T_{2}\left[\sum_{j=1}^{d}\left(R_{1}^{j}\right)^{*}\left(\partial_{j} \psi\right)\right]\right\|_{p, \gamma_{d}} \\
& \leq A_{p}\left\|\sum_{j=1}^{d}\left(R_{1}^{j}\right)^{*}\left(\partial_{j} \psi\right)\right\|_{p, \gamma_{d}} \\
& \leq C_{p} \sum_{j=1}^{d}\left\|\partial_{j} \psi\right\|_{p, \gamma_{d}} .
\end{aligned}
$$

Since $f \in L_{s-1}^{p}\left(\gamma_{d}\right) \cap \mathcal{P}$ and $\partial_{j} f \in L_{s-1}^{p}\left(\gamma_{d}\right) \cap \mathcal{P}$, for each $j=1, \ldots, d$, according to (3.5) with $g=\psi$ we have that

$$
T_{0}\left(\partial_{j} f\right)=(I-L)^{-(s-1) / 2} \partial_{j} \psi
$$

and consequently,

$$
\begin{aligned}
\left\|\partial_{j} \psi\right\|_{p, \gamma_{d}} & =\left\|\left(T_{0} \circ(I-L)^{(s-1) / 2}\right)\left(\partial_{j} f\right)\right\|_{p, \gamma_{d}} \\
& \leq A_{p}\left\|(I-L)^{(s-1) / 2}\left(\partial_{j} f\right)\right\|_{p, \gamma_{d}} \\
& =C_{p}\left\|\partial_{j} f\right\|_{p, s-1},
\end{aligned}
$$


because $T_{0} \circ(I-L)^{(s-1) / 2}=(I-L)^{(s-1) / 2} \circ T_{0}$. Then, from (3.6) we obtain that

$$
\|f\|_{p, s} \leq B_{p, s}\left(\|f\|_{p, s-1}+\sum_{j=1}^{d}\left\|\partial_{j} f\right\|_{p, s-1}\right) .
$$

In the general case, the density of the polynomials in $L_{s}^{p}\left(\gamma_{d}\right)$ is used.

Remark. In the proof of the Proposition 3.1, if $f$ is a constant function, we can see that $f=\psi=h$. Then, trivially we can write

$$
h=(I-L)^{1 / 2} \psi=T_{1} \psi+\left(D_{1}^{\gamma} \circ T_{2}\right) \psi
$$

because $(I-L)^{1 / 2} \psi=\psi, T_{1} \psi=\psi$ and $T_{2} \psi=0$. This way, we obtain that

$$
\|f\|_{p, s-1}=\|f\|_{p, s} .
$$

The Proposition 2.1 and the Proposition 3.1 let us obtain the following corollary.

Corollary 3.1. Suppose $1<p<\infty$ and $s \geq 1$. Then, $f \in L_{s}^{p}\left(\gamma_{d}\right)$ if and only if $f \in L_{s-1}^{p}\left(\gamma_{d}\right)$ and for each $j=1, \ldots, d, \partial_{j} f \in L_{s-1}^{p}\left(\gamma_{d}\right)$. Moreover, the two norms, $\|f\|_{p, s}$ and $\|f\|_{p, s-1}+\sum_{j=1}^{d}\left\|\partial_{j} f\right\|_{p, s-1}$, are equivalent.

Also, by means of the Proposition 3.1 we get the following result.

Corollary 3.2. Given $1<p<\infty$ and $k \geq 1$. Suppose $f \in W_{k}^{p}\left(\gamma_{d}\right)$, then there exists a positive constant $A_{p, k}$ such that

$$
\|f\|_{p, k} \leq A_{p . k}\left(\|f\|_{p, \gamma_{d}}+\sum_{|\alpha| \leq k}\left\|\partial^{\alpha} f\right\|_{p, \gamma_{d}}\right) .
$$

Proof. By using the Proposition 3.1, with $s=1$, we have

$$
\|f\|_{p, 1} \leq B_{p, 1}\left(\|f\|_{p, \gamma_{d}}+\sum_{j=1}^{d}\left\|\partial_{j} f\right\|_{p, \gamma_{d}}\right) .
$$

If $s=2$ we can see that

$$
\|f\|_{p, 2} \leq B_{p, 2}\left(\|f\|_{p, 1}+\sum_{i=1}^{d}\left\|\partial_{i} f\right\|_{p, 1}\right)
$$


and for each $i=1, \ldots, d$,

$$
\left\|\partial_{i} f\right\|_{p, 1} \leq A_{p, 1}\left(\left\|\partial_{i} f\right\|_{p, \gamma_{d}}+\sum_{j=1}^{d}\left\|\partial_{i j}^{2} f\right\|_{p, \gamma_{d}}\right) .
$$

Then by means of the estimates above, we get

$$
\|f\|_{p, 2} \leq C_{p, 2}\left(\|f\|_{p, \gamma_{d}}+\sum_{j=1}^{d}\left\|\partial_{j} f\right\|_{p, \gamma_{d}}+\sum_{i=1}^{d} \sum_{j=1}^{d}\left\|\partial_{i j}^{2} f\right\|_{p, \gamma_{d}}\right),
$$

and the result of this corollary is obtained by induction.

Therefore, we can conclude that

$$
W_{k}^{p}\left(\gamma_{d}\right) \subseteq L_{k}^{p}\left(\gamma_{d}\right) \quad \text { for each } \quad k \in \mathbb{N}
$$

and in consequence, by using (3.3) and (3.7) we obtain the following theorem which it is the principal result of this paper.

Theorem 3.1. Suppose $k$ is a positive integer and $1<p<\infty$. Then

$$
L_{k}^{p}\left(\gamma_{d}\right)=W_{k}^{p}\left(\gamma_{d}\right)
$$

Particularly, as a final comment we observe that

Remark. If $0<s<1,1<p<\infty$ and $k \geq 1$, then $W_{k}^{p}\left(\gamma_{d}\right) \subset L_{s}^{p}\left(\gamma_{d}\right)$ and the inclusion is proper.

In fact, if $0<s<1$ and $k \geq 1$, the inclusion properties of the potential spaces allows us to write

$$
W_{k}^{p}\left(\gamma_{d}\right)=L_{k}^{p}\left(\gamma_{d}\right) \subset L_{s}^{p}\left(\gamma_{d}\right) .
$$

Now, let us consider $d=1, k=1, p=2,0<s<1$ and the function $f$ defined as

$$
f(x)= \begin{cases}\sqrt{x} & \text { if } x \geq 0 \\ 0 & \text { if } x<0\end{cases}
$$

First, one checks that $f \in L^{2}\left(\gamma_{1}\right)$ but $f^{\prime} \notin L^{2}\left(\gamma_{1}\right)$ and therefore $f \notin W_{1}^{2}\left(\gamma_{1}\right)$.

Now, for each $s \in(0,1)$ we define the family of functions

$$
g_{s}(x)= \begin{cases}\sqrt{2^{s} x} & \text { if } x \geq 0 \\ 0 & \text { if } x<0\end{cases}
$$


and from (2.2) we get that the semigroup, $T_{t} g_{s}$, can be written as

$$
T_{t} g_{s}(x)=\frac{\sqrt{2^{s}}}{\sqrt{\pi\left(1-e^{-2 t}\right)}} \int_{0}^{\infty} e^{-\frac{\left|y-e^{-t} x\right|^{2}}{1-e^{-2 t}}} \sqrt{y} d y .
$$

By using the change of variable $u=\frac{y-e^{-t} x}{\sqrt{1-e^{-2 t}}}$ in the above identity, we have that

$$
\begin{aligned}
T_{t} g_{s}(x) & =\sqrt{\frac{2^{s}}{\pi}} \int_{-\frac{x e^{-t}}{\left(1-e^{-2 t}\right)^{1 / 2}}}^{\infty}\left(\sqrt{1-e^{-2 t}} u+e^{-t} x\right)^{1 / 2} e^{-u^{2}} d u \\
& \geq e^{-t / 2} \sqrt{x} \sqrt{\frac{2^{s}}{\pi}} \int_{0}^{\infty} e^{-u^{2}} d u,
\end{aligned}
$$

so, $T_{t} g_{s}(x) \geq 2^{s / 2} e^{-t / 2} \sqrt{x}$, for all $t>0$ and $x \geq 0$. Therefore,

$$
\begin{aligned}
(I-L)^{-s / 2} g_{s}(x) & =\frac{1}{\Gamma(s / 2)} \int_{0}^{\infty} t^{\frac{s}{2}-1} e^{-t} T_{t} g_{s}(x) d t \\
& \geq 2^{s / 2} \sqrt{x}\left(\frac{1}{\Gamma(s / 2)} \int_{0}^{\infty} t^{\frac{s}{2}-1} e^{-2 t} d t\right)
\end{aligned}
$$

and then, by use of the change of variable $t=u / 2$, we obtain

$$
(I-L)^{-s / 2} g_{s}(x) \geq f(x) \text { for each } x \geq 0 .
$$

From (2.17) if $h_{1} \geq h_{2}$, we have that

$$
(I-L)^{-s / 2} h_{1} \geq(I-L)^{-s / 2} h_{2},
$$

because $T_{t}$ is linear and $T_{t} h \geq 0$, if $h \geq 0$. Also, if $h_{1} \geq h_{2}$, we can see that

$$
(I-L)^{s / 2} h_{1} \geq(I-L)^{s / 2} h_{2} \text {. }
$$

In fact, suppose that $h_{1} \geq h_{2}$ but $(I-L)^{s / 2} h_{1}<(I-L)^{s / 2} h_{2}$, then by using (3.9) we obtain a contradiction. Thus, by applying the operator $(I-L)^{s / 2}$ in (3.8), we get

$$
g_{s}(x) \geq(I-L)^{s / 2} f(x)
$$

and consequently,

$$
\|f\|_{2, s}=\left\|(I-L)^{s / 2} f\right\|_{2, \gamma_{1}} \leq\left\|g_{s}\right\|_{2, \gamma_{1}}=2^{s / 2}\|f\|_{2, \gamma_{1}} \leq \sqrt{2}\|f\|_{2, \gamma_{1}},
$$

since $s \in(0,1)$. Therefore, we can conclude that $f \in L_{s}^{2}\left(\gamma_{1}\right)$.

This way, we have obtained a function $f$, such that, $f \notin W_{1}^{2}\left(\gamma_{1}\right)$ but $f \in L_{s}^{2}\left(\gamma_{1}\right)$, if $0<s<1$. 


\section{REFERENCES}

[1] B. Bongionnni, J. L. Torrea, Sobolev spaces associated to the harmonic oscillator, Proc. Indian Acad. Sci. Math. Sci. 116 (3) (2006), 337-360.

[2] B. Bongionnni, J. L. TorReA, What is a Sobolev space for Laguerre function systems?, Studia Math. 192 (2) (2009), 147-172.

[3] L. Forzani, R. Scotto, W. O. Urbina, Riesz and Bessel potentials, the $g^{k}$ functions and an area function for the Gaussian measure $\gamma$, Rev. Un. Mat. Argentina 42 (1) (2000), 17-37.

[4] C. GutiÉrRez, On the Riesz transforms for the Gaussian measures, J. Funct. Anal. 120 (1) (1994), 107-134.

[5] P. Graczyk, J.-J Loeb, I. A. López, A. Nowak, W. O. Urbina, Higher order Riesz transforms, fractional derivates, and Sobolev spaces for Laguerre expansions, J. Math. Pures Appl. 84 (3) (2005), 375-405.

[6] A. A. Kilbas, H. M. Srivastava, J. J. Trujillo, "Theory and Applications of Fractional Differential Equations", North-Holland Mathematics Studies 204, Elsevier Science B.V., Amsterdam, 2006.

[7] I. A. López, W. O. Urbina, Fractional differentiation for the Gaussian measure and applications, Bull. Sci. Math. 128 (7) (2004), 587-603.

[8] I. A. LóPEz, Operators associated with the Jacobi semigroup, J. Approx. Theory 161 (1) (2009), 385-410.

[9] P. A. MEYer, Wuelques résultats analytiques sur le semi-groupe d'OrnsteinUhlenbeck en dimension infinie, in "Theory and Application of Random Fields", Lect. Notes Control Inf. Sci. 49, Springer, Berlin, 1983, 201-214.

[10] P. A. Meyer, Transformations de Riesz pour les lois gaussiennes, in "Seminar of Probability XVIII", Lecture Notes in Math. 1059, Springer, Berlin, 1984, 179-193.

[11] G. PIsIER, Riesz transforms: a simpler analytic proof of P. A. Meyer's inequality, in "Seminar of Probability XXII", Lecture Notes in Math. 1321, Springer, Berlin, 1988, 485-501.

[12] B. Ross, The development of fractional calculus 1695-1900, Historia Math. 4 (1977), 75-89.

[13] P. SJÖGren, Operators associated with the Hermite semigroup - A survey, $J$. Fourier Anal. Appl. 3 (1997), 813-823.

[14] E. M. Stein, "Topics in Harmonic Analysis Related to the Littlewood-Paley Theory", Annals of Mathematics Studies 63, Princeton University Press, Princeton, N. J., 1970.

[15] E. Stein, "Singular Integrals and Differentiability Properties of Functions", Princeton Mathematical Series 30, Princeton University Press, Princeton, N. J., 1970.

[16] H. Sugita, Sobolev spaces of Wiener functionals and Malliavin's calculus, $J$. Math. Kyoto Univ. 25 (1) (1985), 31-48. 
[17] S. Watanabe, "Lectures on Stochastic Differential Equations and Malliavin Calculus", Notes by M. Gopalan Nair and B. Rajeev, Tata Institute of Fundamental Research Lectures on Mathematics and Physics 73, Springer-Verlag, Berlin, 1984.

[18] G. SzegÖ, "Orthogonal Polynomials", American Mathematical Society Colloquium Publications 23, Amer. Math. Soc., Providence, R.I., 1959. 
